\title{
Corrigendum
}

\section{What popular bars post on social media platforms: \\ a case for improved alcohol advertising regulation}

This corrigendum is being published to add a missing author to the following article:

Paradis C, Zhao J, Stockwell T. What popular bars post on social media platforms: a case for improved alcohol advertising regulation. Health Promot Chronic Dis Prev Can. 2020;40(5/6):143-52. https://doi.org/10.24095/hpcdp.40.5/6.03

The two senior authors of this paper (CP and TS) would like to correct the authorship list of this paper to include Sasha Joy-Goatley, and so give proper acknowledgment of her involvement in its early design and implementation of data collection at the first University site. We apologise to her for this omission. The correct citation for the paper now reads:

Paradis C, Zhao J, Joy-Goatley S, Stockwell T. What popular bars post on social media platforms: a case for improved alcohol advertising regulation. Health Promot Chronic Dis Prev Can. 2020;40(5/6):143-52. https://doi.org/10.24095/hpcdp.40.5/6.03

\section{Before correction}

Catherine Paradis, PhD (1); Jinhui Zhao, PhD (2); Tim Stockwell, PhD (2)

\section{After correction}

Catherine Paradis, PhD (1); Jinhui Zhao, PhD (2); Sasha Joy-Goatley, BA (3); Tim Stockwell, PhD (2)

${ }^{3}$ University of Victoria, Victoria, British Columbia, Canada

The original online version and the PDF version of the article have been modified on January 13, 2021, to reflect this change. The original PDF version of the article is available upon request to the editorial team. The records in PubMed, PubMed Central, DOAJ and other full-text repositories have also been modified to reflect this change. 